\title{
Moment-based constrained optimal control of an array of wave energy converters
}

\author{
Nicolás Faedo ${ }^{\mathrm{a}}$, Giordano Scarciotti ${ }^{\mathrm{b}}$, Alessandro Astolfi ${ }^{\mathrm{b}, \mathrm{c}}$ and John V. Ringwood
}

\begin{abstract}
The roadmap to a successful commercialisation of wave energy inherently incorporates the concept of an array or farm of Wave Energy Converters (WECs). These interacting hydrodynamic structures require an optimised process that can ensure the maximum extraction of time-averaged energy from ocean waves, while respecting the physical limitations of each device and actuator characteristics. Recently, a novel optimal control framework based on the concept of moment, for a single WEC device, has been introduced in [1]. Such a strategy offers an energy-maximising computationally efficient solution that can systematically incorporate state and input constraints. This paper presents the mathematical extension of the optimal control framework of [1] to the case where an array of WECs is considered, providing an efficient solution that exploits the hydrodynamic interaction between devices to maximise the total absorbed energy.
\end{abstract}

\section{INTRODUCTION}

Wave energy technology has undergone a strong development over the past years, in line with a growing interest from both the academic and industrial communities in the progress of such a vast renewable energy field. Though the energy from ocean waves has one of the highest power densities [2], the current high installation, operation, maintenance, and decommissioning costs are hindering these novel wave energy extraction technologies to reach a commercialisation stage. As discussed in [3], the roadmap to a successful commercialisation of Wave Energy Converters (WECs) naturally embodies the development of so-called WEC arrays or farms, which effectively incorporates several devices in a common sea area. Hence, any realistic effort to commercialise a novel WEC technology requires both a single WEC and a WEC farm development process.

To further reduce the levelised cost of energy, it is wellknown that WECs require an optimised process that ensures extracting the maximum time-averaged power from ocean waves [4]. Moreover, any realistic formulation must take into account the inherent physical limitations of both the device and the Power Take-Off (PTO) system (actuator) such that the energy extraction is maximised while minimising the risk of component damage. A pioneering yet unconstrained approach to the control of WEC farms can be found in the early study [5]. Not far in time from this publication,

\footnotetext{
aNicolás Faedo and John V. Ringwood are with the Centre for Ocean Energy Research, Maynooth University, Maynooth, Ireland nicolas. faedo.2017@mumail.ie

${ }^{\mathrm{b}}$ Giordano Scarciotti and Alessandro Astolfi are with the Department of Electrical and Electronic Engineering, Imperial College London, London SW7 2AZ, U.K

${ }^{\mathrm{c}}$ Alessandro Astolfi is with the Department of Civil Engineering and Computer Science Engineering, University of Rome “Tor Vergata”, 00133 Rome, Italy
}

[6] incorporates constraints in the motion (amplitude and velocity) of the device, providing a first practical result on constrained control of arrays. More contemporary studies, such as [7], utilise direct transcription techniques to solve for the optimal control law under a numerical scheme (the reader is referred to [4] for a comprehensive list of optimal control strategies considered for this application).

Recently, a novel energy-maximising optimal control framework for a single WEC has been presented in [1]. This formalism is based on the concept of moment (discussed in Section II), and allows mapping the original optimal control problem into a concave Quadratic Program (QP). Then, a computationally efficient calculation of the optimal control law is achieved by state-of-the-art QP solvers [8]. Though [1] accomplishes the energy-maximising objective subject to motion constraints, the mathematical formalism considers only the single-input, single-output (SISO) case, hindering the application of the strategy to the case where an array of WECs is considered. Note that, though one can apply the SISO case of [1] to each isolated device of the array (ignoring the interactions between the WECs composing the farm), this has been already shown to be suboptimal in [7]. In fact, [7] shows that a significant improvement in power absorption can be achieved by considering the corresponding hydrodynamic interactions between devices in the control problem.

Following the roadmap for a successful WEC commercialisation, and given the desirable properties of the momentbased strategy, we present an extension of the moment-based energy-maximising optimal control framework of [1] to the multiple-input, multiple-output (MIMO) case, for which the hydrodynamic interactions between devices are exploited to extract the maximum energy from a given wave field, subject to both state and input constraints. We show that the desirable properties of the moment-based strategy discussed in [1] are retained in the WEC array case.

The remainder of this paper is organised as follows. Section II discusses key concepts behind the moment-matching framework for both SISO and MIMO systems. Section III formally introduces the energy-maximising problem for arrays, while Section IV details the moment-based analysis of the constrained optimal control formulation. Finally, Section $\mathrm{V}$ discusses an application case for a particular array layout, whilst Section VI encompasses the main conclusions of this study. Note that, for brevity, we do not present the proofs of the propositions and lemmas proposed in this paper. These are intended to be presented (along with complementary theoretical results) in an extended version of this paper. 


\section{A. Notation and Preliminaries}

Standard notation is considered through this study, with any exceptions detailed in this section. $\mathbb{R}^{+}\left(\mathbb{R}^{-}\right)$denotes the set of non-negative (non-positive) real numbers. $\mathbb{C}^{0}$ denotes the set of pure-imaginary complex numbers and $\mathbb{C}^{-}$denotes the set of complex numbers with negative real part. The symbol 0 stands for any zero element, dimensioned according to the context. The notation $\mathbb{N}_{q}$ indicates the set of all positive natural numbers up to $q$, i.e. $\mathbb{N}_{q}=\{1,2, \ldots, q\}$. The symbol $\mathbb{I}_{n}$ denotes the identity matrix of dimension $n \times n$, while the notation $\mathbf{1}_{n \times m}$ is used to denote a $n \times m$ Hadamard identity matrix (i.e. a $n \times m$ matrix with all its entries equal to 1). The spectrum of a matrix $A \in$ $\mathbb{R}^{n \times n}$, i.e. the set of its eigenvalues, is denoted as $\lambda(A)$. The symbol $\bigoplus$ denotes the direct sum of $n$ matrices, i.e. $\bigoplus_{i=1}^{n} A_{i}=\operatorname{diag}\left(A_{1}, A_{2}, \ldots, A_{n}\right)$. The vectorisation of a matrix $M \in \mathbb{R}^{n \times m}$ is denoted as $\operatorname{vec}\{M\} \in \mathbb{R}^{n m}$. The Kronecker product between two matrices $M_{1} \in \mathbb{R}^{n \times m}$ and $M_{2} \in \mathbb{R}^{p \times q}$ is denoted as $M_{1} \otimes M_{2} \in \mathbb{R}^{n p \times m q}$, while the Kronecker sum is denoted as $M_{1} \hat{\oplus} M_{2}$. The Kronecker delta function is denoted as ${ }_{j}^{i} \delta$. The convolution between two functions $f(t)$ and $g(t)$ over the finite range $[0, t]$, i.e. $\int_{0}^{t} f(\tau) g(t-\tau) d \tau$ is denoted as $f * g$. The set of all realvalued square integrable functions is denoted as $L^{2}(\mathbb{R})$. The symbol $e_{i j}^{q} \in \mathbb{R}^{q \times q}$ denotes a matrix with 1 in the $i j$ entry and 0 elsewhere. The symbol $\varepsilon_{n} \in \mathbb{R}^{n \times 1}$ denotes a vector with odd entries equal to 1 and even entries equal to 0 .

\section{MOMENT-BASED THEORY}

In this section we briefly recall some of the key concepts behind the moment-matching framework, as in [9], [10].

Consider a finite-dimensional, SISO, continuous-time system described, for $t \geq 0$, by the state-space model

$$
\dot{x}(t)=A x(t)+B u(t), \quad y(t)=C x(t)
$$

where $x(t) \in \mathbb{R}^{n}, u(t) \in \mathbb{R}, y(t) \in \mathbb{R}, A \in \mathbb{R}^{n \times n}, B \in \mathbb{R}^{n}$ and $C \in \mathbb{R}^{1 \times n}$ and assume that (1) is minimal.

Lemma 1: [9], [10] Consider system (1) and the autonomous signal generator

$$
\dot{\xi}(t)=S \xi(t), \quad u(t)=L \xi(t),
$$

with $\xi(t) \in \mathbb{R}^{\nu}, S \in \mathbb{R}^{\nu \times \nu}, L \in \mathbb{R}^{1 \times \nu}$ and $\xi(0) \in \mathbb{R}^{\nu}$. Assume that the triple $(L, S, \xi(0))$ is minimal, $\lambda(A) \subset \mathbb{C}^{-}$, $\lambda(S) \subset \mathbb{C}^{0}$ and the eigenvalues of $S$ are simple. Then, there is a unique matrix $\Pi \in \mathbb{R}^{n \times \nu}$ which solves the Sylvester equation

$$
A \Pi+B L=\Pi S
$$

and the steady-state response of the output of the interconnected system (1)-(2) is $y_{s s}(t)=C \Pi \xi(t)$.

Remark 1: The minimality of the triple $(L, S, \xi(0))$ implies the observability of the pair $(L, S)$ and the excitability ${ }^{1}$ of the pair $(S, \xi(0))$.

\footnotetext{
${ }^{1}$ We refer the reader to [10] for the definition of excitability.
}

Definition 1: The matrix $C \Pi$, with $\Pi$ solution of the Sylvester equation (3), is the moment of system (1) at the signal generator (2).

Remark 2: From now on, we refer to the matrix $\underline{Y}=C \Pi$ as the moment-domain equivalent of $y(t)$.

\section{A. MIMO case}

We now consider a finite-dimensional, MIMO, continuoustime system described, for $t \geq 0$, by the state-space model

$$
\dot{x}(t)=A x(t)+B u(t), \quad y(t)=C x(t),
$$

with $^{2} x(t) \in \mathbb{R}^{n}, u(t) \in \mathbb{R}^{q}, y(t) \in \mathbb{R}^{q}, A \in \mathbb{R}^{n \times n}, B \in$ $\mathbb{R}^{n \times q}, C \in \mathbb{R}^{q \times n}$ and assume that (4) is minimal.

In the following, we present an adaptation of Lemma 1 for the MIMO case.

Lemma 2: Let each input $u_{i}(t)$ of system (4), with $u(t)=$ $\left[u_{1}(t), \ldots, u_{q}(t)\right]^{\top}$, be generated by the autonomous, singleoutput signal generator ${ }^{3}$

$$
\dot{\xi}(t)=S \xi(t), \quad u_{i}(t)=L_{i} \xi(t),
$$

with $\xi(t) \in \mathbb{R}^{\nu}$ and $L_{i} \in \mathbb{R}^{1 \times \nu}$. Assume that the pair $\left(L_{i}, S\right)$ is observable for all $i \in \mathbb{N}_{q}, \lambda(A) \subset \mathbb{C}^{-}$, and $S$ is as in Lemma 1. Consider the autonomous multiple-output signal generator

$$
\dot{\Xi}(t)=\left(\mathbb{I}_{q} \otimes S\right) \Xi(t), \quad u(t)=\left(\sum_{i=1}^{q} e_{i i}^{q} \otimes L_{i}\right) \Xi(t),
$$

$\Xi(t) \in \mathbb{R}^{q \nu}$ and assume that the pair $\left(\left(\mathbb{I}_{q} \otimes S\right), \Xi(0)\right)$ is excitable. Then, there is a unique matrix $\Pi \in \mathbb{R}^{n \times q \nu}$ which solves the Sylvester equation

$$
A \Pi-\Pi\left(\mathbb{I}_{q} \otimes S\right)=-B\left(\sum_{i=1}^{q} e_{i i}^{q} \otimes L_{i}\right) .
$$

and the steady-state response of the output of the interconnected system is $y_{s s}(t)=C \Pi \Xi(t)$.

Remark 3: Analogously to the SISO case, the moment for system (4) is computed in terms of the unique solution of a Sylvester equation, i.e. equation (7).

\section{ENERGY-MAXIMISING CONTROL FORMULATION}

The control problem for an array of $N$ devices can be informally posed as follows: compute the optimal control input (PTO force) acting on each device $u_{i}(t)$ such that the time-averaged energy absorbed by the (complete) WEC array is maximised over a time interval of length $T$. To state this energy-maximising criterion in terms of an objective function, we note that the useful energy converted by the totality of the PTOs composing the array can be computed as

$$
\mathcal{J}=\sum_{i=1}^{N} \frac{1}{T} \int_{0}^{T} u_{i}(\tau) \dot{x}_{i}(\tau) d \tau=\frac{1}{T} \int_{0}^{T} P(\tau) d \tau,
$$

\footnotetext{
${ }^{2}$ We focus on square systems, motivated by the WEC application.

${ }^{3}$ Although we assume the same dynamic matrix $S$ for all $u_{i}$ to simplify the notation, each input can be driven by an independent signal generator, i.e. $\dot{\xi}_{i}=S_{i} \xi_{i}, u_{i}=L_{i} \xi_{i}$.
} 
where $\dot{x}_{i}$ and $P$ denote the velocity of the $i$-th device and the total instantaneous power of the WEC array, respectively. The calculation of such an optimal control law is non-trivial, mainly due to the irregularity of the poly-chromatic input (the wave excitation force) to the system.

\section{A. Equations of motion of an array of WECs}

This section introduces the basics behind linear modelling of arrays of wave energy converters in the time-domain. The modelling assumptions considered are consistent across a wide variety of WEC control and estimation applications presented in the literature, as detailed in [4].

The linearised equation of motion for an array of $N$ wave energy converters can be expressed in time-domain according to Newton's second law, obtaining the linear hydrodynamic formulation:

$$
M \ddot{\chi}(t)=\mathcal{F}_{r}(t)+\mathcal{F}_{h}(t)+\mathcal{F}_{e}(t)-\mathcal{U}(t),
$$

where $M=\bigoplus_{i=1}^{N} m_{i}$ is the mass matrix of the buoy with $m_{i}$ the mass of the $i$-th device, and each element of the vectors $\chi, \mathcal{F}_{e}, \mathcal{F}_{h}, \mathcal{F}_{r} \in \mathbb{R}^{N}$ contain the excursion $x_{i}(t)$, the excitation force $f_{e_{i}}(t)$, the hydrostatic restoring force $f_{h_{i}}(t)$ and the radiation force $f_{r_{i}}(t)$ acting on the $i$-th device $(i \in$ $\mathbb{N}_{N}$ ) of the array, respectively. The vector $\mathcal{U}(t)$ is composed of the PTO forces exerted on each device $u_{i}(t)$.

The linearised hydrostatic force $\mathcal{F}_{h}(t)$ can be written as $-S_{h} \chi(t)$, where $S_{h}=\bigoplus_{i=1}^{N} s_{h_{i}}$ and each $s_{h_{i}}>0$ denotes the hydrostatic stiffness of the $i$-th WEC. The radiation force $\mathcal{F}_{r}(t)$ is modelled from linear potential theory and, using Cummins' equation [11], is

$$
\mathcal{F}_{r}(t)=-\mu_{\infty} \ddot{\chi}(t)-\int_{0}^{+\infty} K(\tau) \dot{\chi}(t-\tau) d \tau,
$$

where $^{4} \mu_{\infty}=\lim _{\omega \rightarrow+\infty} \tilde{A}(\omega), \mu_{\infty}>0$ represents the added-mass matrix at infinite frequency and $K(t)=$ $\sum_{i=1}^{N} \sum_{j=1}^{N} e_{i j}^{N} \otimes k_{i j}(t) \in \mathbb{R}^{N \times N}, k_{i j}(t) \in L^{2}(\mathbb{R})$, contains the (causal) radiation impulse response of each device (if $i=j$ ) and each interaction due to the radiated waves created by the motion of other devices (if $i \neq j$ ). The linearised equation of motion of the WEC can be expressed as

$$
\left(M+\mu_{\infty}\right) \ddot{\chi}(t)+K(t) * \dot{\chi}(t)+S_{h} x(t)=\mathcal{F}_{e}(t)-\mathcal{U}(t) .
$$

The system described by the Volterra integro-differential equation (11), for the WEC case is internally stable and strictly passive with respect to the output, for any physically meaningful values of the parameters and the mapping $K(t)$ involved, see [12].

\section{B. Optimal control formulation and motion constraints}

As discussed previously in Section I, any optimal control approach should consider both motion and actuator constraints, since the unconstrained optimal solution that maximises energy absorption (in the monochromatic input case) is often unrealistic in terms of displacement and PTO force requirements [4], [12].

\footnotetext{
${ }^{4}$ See [12] for the definition of $\tilde{A}(\omega)$.
}

We consider herein constraints on the displacement and velocity of each WEC composing the array, $x_{i}(t)$ and $\dot{x}_{i}(t)$, respectively, and each PTO force $u_{i}(t)$, which can be compactly written, for all $i \in \mathbb{N}_{N}$, as

$$
\left\{\begin{array}{l}
\left|x_{i}(t)\right| \leq X_{\max }, \\
\left|\dot{x}_{i}(t)\right| \leq V_{\max }, \\
\left|u_{i}(t)\right| \leq U_{\max }
\end{array} \quad \forall t \in \mathbb{R},\left(X_{\max }, V_{\max }, U_{\max }\right) \in \mathbb{R}^{+{ }^{3}} .\right.
$$

Given the objective function defined in (8), the governing dynamics of the WEC array in (11) and the set of motion and input constraints defined in (12), the energy-maximising optimal control problem can be stated as

$$
\begin{aligned}
\mathcal{U}^{\mathrm{opt}}(t) & =\arg \max _{\mathcal{U}(t)} \mathcal{J} \\
& \text { subject to: } \\
& \left\{\begin{array}{l}
\text { WEC array dynamics }(11), \\
\text { Motion constraints (12) }
\end{array}\right.
\end{aligned}
$$

\section{MOMEnT-BASED WEC ARRAY FORMULATION}

To use the theoretical framework recalled in Section II on this WEC array case we need to re-write the equation of motion presented in (11) in a suitable structure. The following state-space representation is therefore proposed:

$$
\dot{\varphi}(t)=A_{\varphi} \varphi(t)+B_{\varphi} \mathfrak{u}(t), \quad y_{\varphi}(t)=C_{\varphi} \varphi(t)=\dot{\chi}(t),
$$

where $\varphi(t)=\left[\phi_{1}, \ldots, \phi_{N}\right]^{\top} \in \mathbb{R}^{2 N}$ is the state-vector of the continuous-time model, with $\phi_{i}=\left[x_{i}(t), \dot{x}_{i}(t)\right]^{\top}$. The function $\mathfrak{u}(t) \in \mathbb{R}^{N}$, assumed to be the input to the system (14), is defined as

$$
\mathfrak{u}(t)=\mathcal{F}_{e}(t)-K(t) * \dot{\chi}(t),
$$

Under this assumption, the matrices in (14) can be written, in compact form, as

$$
\begin{aligned}
A_{\varphi} & =\sum_{i=1}^{N} \sum_{j=1}^{N} e_{i j}^{N} \otimes A_{\varphi_{i j}}, B_{\varphi}=\sum_{i=1}^{N} \sum_{j=1}^{N} e_{i j}^{N} \otimes B_{\varphi_{i j}} \\
C_{\varphi} & =\mathbb{I}_{N} \otimes[01],
\end{aligned}
$$

with each $A_{\varphi_{i j}} \in \mathbb{R}^{2 \times 2}, B_{\varphi_{i j}} \in \mathbb{R}^{2}$ defined as

$$
A_{\varphi_{i j}}=\left[\begin{array}{cc}
0 & { }_{j}^{i} \delta \\
-\mathcal{M}_{i j} s_{h_{i}} & 0
\end{array}\right], B_{\varphi_{i j}}=\left[\begin{array}{c}
0 \\
\mathcal{M}_{i j}
\end{array}\right],
$$

where $\mathcal{M}_{i j}$ is the $i j$-th element of the inverse generalised mass matrix $\left(M+\mu_{\infty}\right)^{-1}$.

Within the moment-based framework, each $i$-th entry of the vectors $\mathcal{F}_{e}$ and $\mathcal{U}$ are expressed as the output of the signal generators

$$
\dot{\xi}_{i}(t)=S \xi_{i}(t), f_{e_{i}}(t)=L_{e_{i}} \xi_{i}(t), u_{i}(t)=L_{u_{i}} \xi_{i}(t),
$$

where the dimension of $S, L_{e_{i}}$ and $L_{u_{i}}$ are as in (2), $\xi_{i}(t) \in$ $\mathbb{R}^{\nu}$ and the pairs $\left(L_{e_{i}}, S\right)$ and $\left(L_{u_{i}}, S\right)$ are observable. Given the characteristics of $\lambda(S)$, we consider the finite set $\mathscr{F}=$ 
$\left\{\omega_{p}\right\}_{p=1}^{f} \subset \mathbb{R}$ and write the matrix $S$ in a real block-diagonal form as

$$
S=\bigoplus_{p=1}^{f}\left[\begin{array}{cc}
0 & \omega_{p} \\
-\omega_{p} & 0
\end{array}\right]
$$

where $\nu=2 f, f \geq 0$ integer. Finally, both the excitation force and control force vectors are expressed as the solution of the autonomous multiple-output signal generator as

$$
\begin{aligned}
\dot{\Xi}(t) & =\left(\mathbb{I}_{N} \otimes S\right) \Xi(t), \\
\mathcal{F}_{e}(t) & =\left(\sum_{i=1}^{N} e_{i i}^{N} \otimes L_{e_{i}}\right) \Xi(t)=L_{e} \Xi(t), \\
\mathcal{U}(t) & =\left(\sum_{i=1}^{N} e_{i i}^{N} \otimes L_{u_{i}}\right) \Xi(t)=L_{u} \Xi(t),
\end{aligned}
$$

where, without loss of generality, the initial condition of the signal generator is chosen as $\Xi_{e}(0)=\varepsilon_{N \nu}$.

With this selection of matrices the moments of system (14), driven by the signal generator (20), can be computed by solving the Sylvester equation (see Lemma 2)

$$
A_{\varphi} \Pi_{\varphi}+B_{\varphi}\left(L_{e}-L_{u}-\underline{\mathcal{Z}}\right)=\Pi_{\varphi}\left(\mathbb{I}_{N} \otimes S\right),
$$

where $\Pi_{\varphi} \in \mathbb{R}^{2 N \times N \nu}$ and $\underline{\mathcal{Z}}$ is the moment-domain equivalent of the radiation matrix convolution term. The momentdomain equivalent of the velocity can be expressed in terms of the solution of (21) straightforwardly as $\underline{\mathcal{V}}=C_{\varphi} \Pi_{\varphi}$. Moreover, the term $\underline{\mathcal{Z}}$ depends on $\Pi_{\varphi}$, hence we cannot yet solve (21). In the following, we first define the quantity $\underline{\mathcal{Z}}$ and we then provide an explicit solution for (21).

Proposition 1: The moment-domain equivalent of the convolution integral in (10) can be computed as

$$
\underline{\mathcal{Z}}=\sum_{i=1}^{N} \sum_{j=1}^{N} e_{i j}^{N} \underline{\mathcal{V}}\left(\mathbb{I}_{N} \otimes \mathscr{R}_{i j}\right),
$$

where each $\mathscr{R}_{i j} \in \mathbb{R}^{\nu \times \nu}$ is a block-diagonal matrix defined as

$$
\mathscr{R}_{i j}=\bigoplus_{p=1}^{f}\left[\begin{array}{cc}
{ }_{j}^{i} r_{\omega_{p}} & { }_{j}^{i} m_{\omega_{p}} \\
-{ }_{j}^{i} m_{\omega_{p}} & { }_{j}^{i} r_{\omega_{p}}
\end{array}\right],
$$

with

$$
{ }_{j}^{i} r_{\omega_{p}}=\tilde{B}\left(\omega_{p}\right)_{i j}, \quad{ }_{j}^{i} m_{\omega_{p}}=\omega_{p}\left[\tilde{A}\left(\omega_{p}\right)_{i j}-\mu_{\infty_{i j}}\right],
$$

where $\tilde{A}(\omega)_{i j}$ is the added-mass matrix, $\tilde{B}(\omega)_{i j}$ is the radiation damping matrix ${ }^{5}$ of the device at each specific frequency induced by the eigenvalues of $S$, and $\mu_{\infty i j}$ is the $i j$-th entry of the matrix $\mu_{\infty}$.

Using the analytical definition of the moment-domain equivalent of the convolution term in (22) we state the following proposition that addresses the explicit computation of the moment equivalent $\underline{\mathcal{V}}$.

Proposition 2: Under the internal stability of (14), the moment-domain equivalent of the output $y_{\varphi}$ of system (14) can be uniquely determined as

$$
\operatorname{vec}\{\underline{\mathcal{V}}\}=\left(\mathbb{I}_{N} \otimes \Phi_{\varphi}^{\mathscr{R}}\right) \operatorname{vec}\left\{L_{e}-L_{u}\right\},
$$

${ }^{5}$ See [12] for the definition of $\tilde{B}(\omega)$. where

$$
\begin{aligned}
& \Phi_{\varphi}^{\mathscr{R}}=\left(\mathbb{I}_{\nu} \otimes C_{\varphi}\right) \Phi_{\varphi}^{-1}\left(\mathbb{I}_{\nu} \otimes B_{\varphi}\right) \\
& \Phi_{\varphi}=\left(S \hat{\oplus} A_{\varphi}\right)+\sum_{i=1}^{N} \sum_{j=1}^{N} \mathscr{R}_{i j}^{\top} \otimes-B_{\varphi} e_{i j}^{N} C_{\varphi},
\end{aligned}
$$

with $\Phi_{\varphi} \in \mathbb{R}^{2 N \nu \times 2 N \nu}$ and $\Phi_{\varphi}^{\mathscr{R}} \in \mathbb{R}^{N \nu \times N \nu}$.

With the result of Proposition 2, we address the formulation of (13) using a moment-based approach. In particular, we show that the objective function $\mathcal{J}$ significantly simplifies under this framework.

Proposition 3: Consider the expression for the instantaneous power $P$ in (8) and the representation for $u_{i}$ as in (18). Define the set $\mathscr{F}$ considered to compute $S$ in (19) as $\mathscr{F}=\left\{p \omega_{0}\right\}_{p=1}^{f}$. Then, the absorbed power $\mathcal{J}$ over the time period $[0, T]$, with $T=2 \pi / \omega_{0}$, can be computed as

$$
\mathcal{J}=\frac{1}{2} \sum_{i=1}^{N} \underline{\mathcal{V}}_{i} L_{u_{i}}^{\top}
$$

where $\underline{\mathcal{V}}_{i}$ denotes the moment-domain equivalent of the velocity of the $i$-th device.

Remark 4: The selection of the set $\mathscr{F}=\left\{p \omega_{0}\right\}_{p=1}^{f}$ is a standard assumption for the numerical generation of ocean waves [13].

Proposition 3 shows that, under our moment-based strategy, the objective function of (8) can be computed as the sum of $N$ inner-product operations in $\mathbb{R}^{1 \times N \nu}$. Furthermore, we show in the following that, under the presented momentbased strategy, the (motion unconstrained ${ }^{6}$ ) optimisation problem of (13) has a strictly concave QP formulation.

Proposition 4: Consider the (motion unconstrained) energy-maximising optimal control problem (13). Then, under the same assumptions of Proposition 3, the optimal control law $\mathcal{U}^{\mathrm{opt}}=L_{u}^{\mathrm{opt}} \Xi$ can be uniquely computed in the moment-domain as

$$
\begin{aligned}
L_{u}^{\mathrm{opt}}=\arg \max _{L_{u}}- & \frac{1}{2} \operatorname{vec}\left\{L_{u}\right\}^{\top}\left(\mathbb{I}_{N} \otimes \Phi_{\varphi}^{\mathscr{R}^{\top}}\right) \operatorname{vec}\left\{L_{u}\right\}+ \\
& \frac{1}{2} \operatorname{vec}\left\{L_{e}\right\}^{\top}\left(\mathbb{I}_{N} \otimes \Phi_{\varphi}^{\mathscr{R}^{\top}}\right) \operatorname{vec}\left\{L_{u}\right\} .
\end{aligned}
$$

Remark 5: Proposition 4 maps the original optimal control formulation of (13) into a standard (concave) QP program, which can be efficiently solved using well-known QP solvers, such as those described in [8].

\section{A. Handling motion constraints in moment-domain}

Following [1], we map the set of motion constraints to their respective moment-domain equivalents ${ }^{7}$, i.e.

$$
\text { Eq. (12) } \mapsto\left\{\begin{array}{l}
\left|\underline{\mathcal{V}}_{i}\left(\mathbb{I}_{N} \otimes S^{-1}\right) e^{\left(\mathbb{I}_{N} \otimes S\right) t} \varepsilon_{N \nu}\right| \leq X_{\max } \\
\left|\underline{\mathcal{V}}_{i} e^{\left(\mathbb{I}_{N} \otimes S\right) t} \varepsilon_{N \nu}\right| \leq V_{\max } \\
\left|L_{u} e^{\left(\mathbb{I}_{N} \otimes S\right) t} \varepsilon_{N \nu}\right| \leq U_{\max } .
\end{array}\right.
$$

\footnotetext{
${ }^{6}$ This refers to the objective function (13) under the assumption that the constraints in the motion of the device defined in (12) are not considered in the formulation.

${ }^{7}$ Note that the moment-domain equivalent of the position $x_{i}(t)$ can be expressed [14] as $\underline{\mathcal{V}}_{i}\left(\mathbb{I}_{N} \otimes S^{-1}\right)$
} 
and we enforce them only at a set of specified time instants (sometimes referred to as collocation points), i.e. $\left\{t_{i}\right\}_{i=1}^{N_{c}}$. Defining the vectors $\Lambda \in \mathbb{R}^{N N_{c} \times N^{2} \nu}$ and $\Delta \in \mathbb{R}^{2 N N_{c} \times N^{2} \nu}$

as

$$
\begin{aligned}
\Lambda & =\left[\left(e^{\left(\mathbb{I}_{N} \otimes S\right) t_{1}} \varepsilon_{N \nu} \otimes \mathbb{I}_{N}\right)^{\top}|\ldots|\left(e^{\left(\mathbb{I}_{N} \otimes S\right) t_{1}} \varepsilon_{N \nu} \otimes \mathbb{I}_{N}\right)^{\top}\right], \\
\Delta & =[\Lambda \mid-\Lambda]
\end{aligned}
$$

and substituting $\underline{\mathcal{V}}$ using (25), the motion constrained energymaxisimising optimal control law can be fully written in moment-domain as an inequality-constrained $\mathrm{QP}$ problem, i.e.:

$$
\begin{aligned}
L_{u}^{\mathrm{opt}}=\arg \max _{L_{u}}- & \frac{1}{2} \operatorname{vec}\left\{L_{u}\right\}^{\top}\left(\mathbb{I}_{N} \otimes \Phi_{\varphi}^{\mathscr{R}^{\top}}\right) \operatorname{vec}\left\{L_{u}\right\}+ \\
& \frac{1}{2} \operatorname{vec}\left\{L_{e}\right\}^{\top}\left(\mathbb{I}_{N} \otimes \Phi_{\varphi}^{\mathscr{R}^{\top}}\right) \operatorname{vec}\left\{L_{u}\right\}
\end{aligned}
$$

subject to:

$$
\begin{aligned}
& \mathcal{A}_{\chi} \operatorname{vec}\left\{L_{u}\right\} \leq \mathcal{B}_{\chi} \\
& \mathcal{A}_{\dot{\chi}} \operatorname{vec}\left\{L_{u}\right\} \leq \mathcal{B}_{\dot{\chi}} \\
& \mathcal{A}_{\mathcal{U}} \operatorname{vec}\left\{L_{u}\right\} \leq \mathcal{B}_{\mathcal{U}}
\end{aligned}
$$

where

$$
\begin{aligned}
& \mathcal{A}_{\chi}=-\Delta\left(\mathbb{I}_{N} \otimes\left(S^{-1} \otimes \mathbb{I}_{N}\right)^{\top} \Phi_{\varphi}^{\mathscr{R}}\right), \\
& \mathcal{B}_{\chi}=X_{\max } \mathbf{1}_{2 N N_{c} \times 1}-\mathcal{A}_{\chi} \operatorname{vec}\left\{L_{e}\right\}, \\
& \mathcal{A}_{\dot{\chi}}=-\Delta\left(\mathbb{I}_{N} \otimes \Phi_{\varphi}^{\mathscr{R}}\right), \\
& \mathcal{B}_{\dot{\chi}}=V_{\max } \mathbf{1}_{2 N N_{c} \times 1}-\mathcal{A}_{\dot{\chi}} \operatorname{vec}\left\{L_{e}\right\}, \\
& \mathcal{A}_{\mathcal{U}}=\Delta, \\
& \mathcal{B}_{\mathcal{U}}=U_{\text {max }} \mathbf{1}_{2 N N_{c} \times 1} .
\end{aligned}
$$

\section{Application CASE}

This section presents an application case to illustrate the proposed strategy based on the square-type WEC array layout studied in [15], and depicted in Figure 1. This farm is

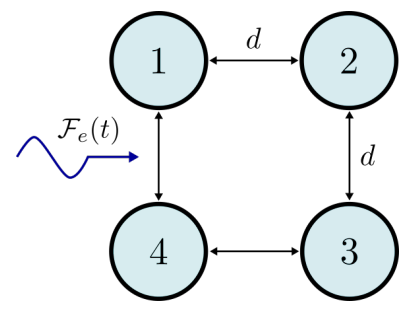

Fig. 1. WEC array layout considered for the application example (adapted from [15]).

composed by four identical cylindrical-type WECs arranged in a square layout with a distance $d=20$ [m] between devices. Each cylindrical body has a diameter of 10 [m], a draft of $10[\mathrm{~m}]$ and a mass of $7.9 \times 10^{5}$ [kg]. For the numerical study of this section we consider a JONSWAP spectrum [16] for the generation of the irregular (polychromatic) waves with a peak period of $T_{p}=10$ [s], significant wave height $H_{s}=3[\mathrm{~m}]$ and peak enhancement factor $\gamma=3.3$. The value for $f$ in (19) is chosen as 30, following a similar analysis to the one proposed in [1]. We consider the following values for the motion constraints: $X_{\max }=1[\mathrm{~m}], V_{\max }=0.5[\mathrm{~m} / \mathrm{s}]$ and $U_{\max }=0.7 \times 10^{6}[\mathrm{~N}]$.

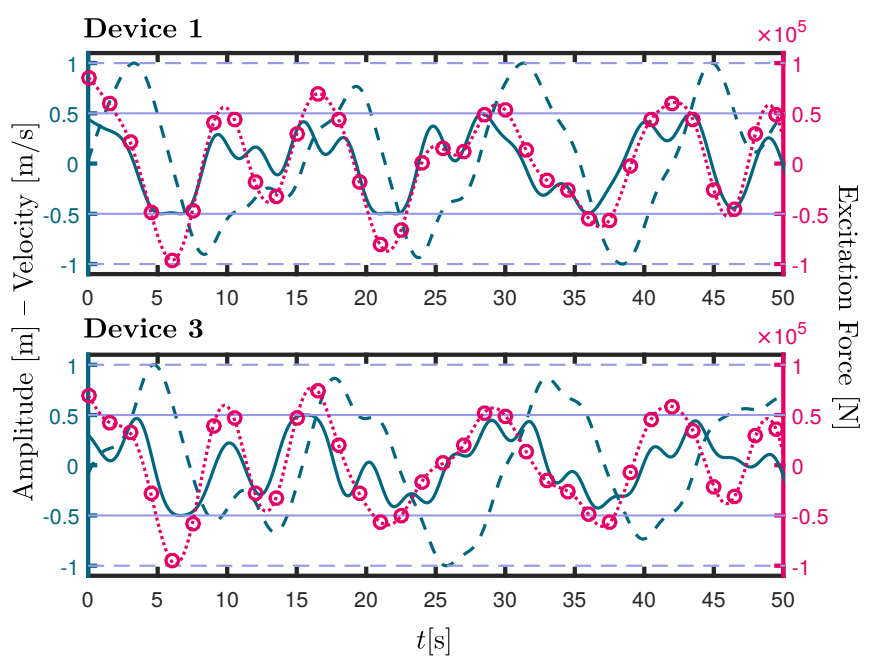

Fig. 2. Motion results for devices 1 and 3 under the proposed momentbased strategy. The left Y-axis (dark green) displays amplitude (dashed line) and velocity (solid line), while the right Y-axis (red) depicts the wave excitation force. The horizontal lines (violet) represent the value of each corresponding motion constraint.

Figure 2 presents the motion results for devices 1 and 3 obtained using the solution of the optimal control formulation in (31). The left Y-axis (dark green) denotes amplitude (dashed line) and velocity (solid line), while the right Y-axis (red) characterises the excitation force values (dotted line, circular marker). The constraint limits for both amplitude, $X_{\max }$, and velocity, $V_{\max }$, are plotted along the time traces with the corresponding line pattern (violet). It can be immediately appreciated that the selected constraints are respected consistently across the entire simulation time, proving an effective handling of motion constraints for this MIMO moment-based strategy. It is also interesting to note that the velocity of the device when applying the computed optimal control law seems to remain "in-phase" 8 with the wave excitation force, consistently with the well-known (unconstrained) theoretical result on maximum power absorption for mono-chromatic waves. Finally, Figure 3 presents the optimal control input computed with our moment-based strategy for device 1 (dashed-dotted line) and device 3 (dotted line), which can also be seen to respect the corresponding user-defined constraint of $0.7 \times 10^{6}[N]$. We note that Devices 2 and 4 present a similar behavior and they are omitted for economy of space

As discussed in Section III-B, the necessity of considering motion constraints stems from the fact that the unconstrained energy-maximising optimal solution often requires unrealistic values for the physical variables of the analysed WEC system. Naturally, constraining the motion of the device leads

\footnotetext{
${ }^{8} \mathrm{We}$ acknowledge that the mathematical concept of phase is no longer defined for a signal with multiple frequency components. In here, we use the term "in-phase" to indicate that the peaks (local maxima and minima) of both signals are aligned in time.
} 


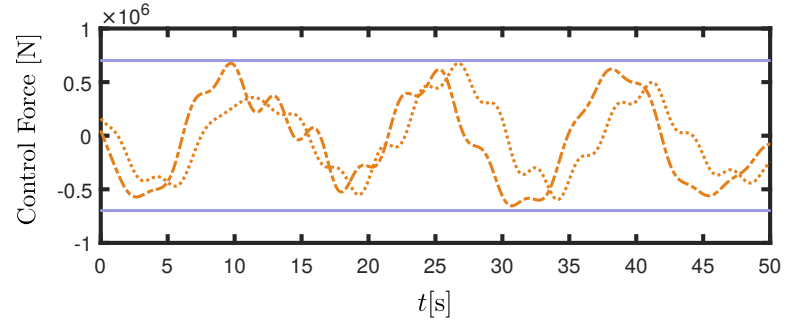

Fig. 3. Optimal control input computed with the proposed momentbased strategy for device 1 (dashed-dotted) and device 3 (dotted line). The horizontal line (violet) represents the force constraint limit.

to a decrease in the total absorbed power. This motivates us to explicitly analyse how the constraints affect the total power absorbed by the WEC farm of Figure 1 when using the moment-based strategy proposed in the paper. To fulfill this objective we consider regular (mono-chromatic) waves of a fixed height of $3[\mathrm{~m}]$ and with different wave periods $T \in$ $[5,10]$. For each of this selected periods, we compute both the optimal unconstrained time-averaged power absorption $\mathcal{J}_{T}^{\text {unc }}$ and the maximum absolute value of the device amplitude i.e., $\max \left|\chi_{T}^{\mathrm{unc}}\right|$. We now propose the definition of a power absorption ratio:

$$
R_{P}=\frac{\mathcal{J}_{T}^{\text {con }, R_{A}}}{\mathcal{J}_{T}^{\text {unc }}}
$$

where $\mathcal{J}_{T}^{\text {con, } R_{A}}$ is the total power absorption for a wave of pe$\operatorname{riod} T$ and amplitude constrained to $X_{\max }=R_{A} \max \left|\chi_{T}^{\text {unc }}\right|$, $R_{A} \in[0,1]$.

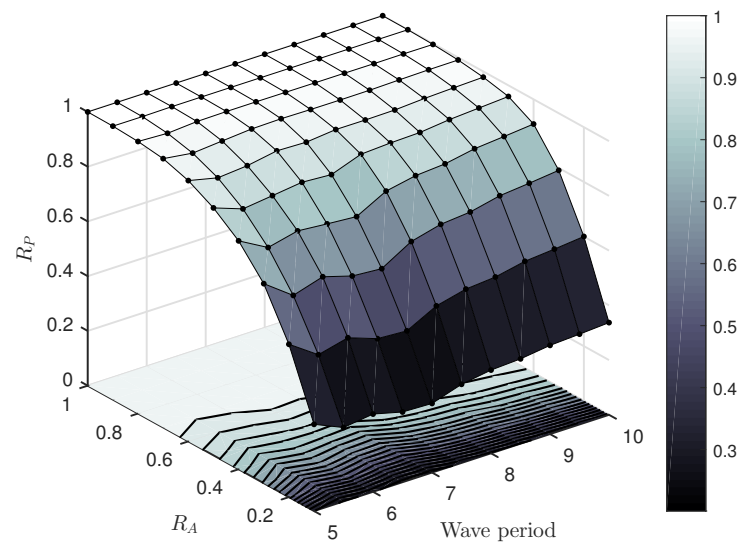

Fig. 4. Power absorption ratio $R_{P}$ for different values of constraint factor $R_{A}$ and wave period $T$.

Figure 4 illustrates the results obtained for $R_{P}$ with varying constraint factor $R_{A}$ and wave period $T$. It is noteworthy that with an amplitude constraint of $40 \%$ of the optimal unconstrained motion, the presented moment-based strategy is capable of extracting more than $\approx 80 \%$ of the unconstrained optimal result for the totality of the analysed periods, being almost $90 \%$ in the case of $T \geq 8$.

\section{CONCLUSiONS}

This study introduces an extension of the moment-based energy-maximising technique developed in [1] to MIMO systems. We show that the desirable properties of the strategy in the SISO case, such as the mapping of the original objective function to a concave QP problem, are also present in this MIMO extension. We have also detailed herein how to handle both state and input constraints in moment-domain for this WEC array case, further exploiting the advantages of the moment-based strategy. Finally, this paper demonstrates the usage of the proposed method by means of a full-scale 4-device WEC farm, showing the potential of this novel mathematical framework to help in the roadmap towards the commercialisation of WEC technologies.

\section{ACKNOWLEDGMENT}

This material is based upon works supported by Science Foundation Ireland under Grant no. 13/IA/1886 and to the Royal Society International Exchange Cost Share programme (IEC $\backslash \mathrm{R} 1 \backslash 180018$ ). This work has been partially supported by the European Union's Horizon 2020 Research and Innovation Programme under grant agreement No 739551 (KIOS $\mathrm{CoE})$.

\section{REFERENCES}

[1] N. Faedo, G. Scarciotti, A. Astolfi, and J. V. Ringwood, "Energymaximising control of wave energy converters using a moment-domain representation," Control Engineering Practice, vol. 81, pp. 85 - 96, 2018.

[2] J. Cruz, Ocean wave energy: current status and future perspectives. Springer Science \& Business Media, 2008.

[3] K. Ruehl and D. Bull, "Wave Energy Development Roadmap: Design to commercialization," OCEANS 2012 MTS/IEEE: Harnessing the Power of the Ocean, 2012.

[4] N. Faedo, S. Olaya, and J. V. Ringwood, "Optimal control, MPC and MPC-like algorithms for wave energy systems: An overview," IFAC Journal of Systems and Control, vol. 1, pp. 37-56, 2017.

[5] D. Evans, "Some theoretical aspects of three-dimensional wave-energy absorbers," in Proceedings of the first symposium on wave energy utilization, Chalmers University of Technology, Gothenburg, Sweden, 1979, pp. 77-106.

[6] G. Thomas and D. Evans, "Arrays of three-dimensional wave-energy absorbers," Journal of Fluid Mechanics, vol. 108, pp. 67-88, 1981.

[7] G. Bacelli, P. Balitsky, and J. V. Ringwood, "Coordinated control of arrays of wave energy devicesbenefits over independent control," IEEE Transactions on Sustainable Energy, vol. 4, no. 4, pp. 1091-1099, 2013.

[8] S. Boyd and L. Vandenberghe, Convex optimization. Cambridge university press, 2004

[9] A. Astolfi, "Model reduction by moment matching for linear and nonlinear systems," IEEE Transactions on Automatic Control, vol. 55 , no. 10, pp. 2321-2336, 2010.

[10] G. Scarciotti and A. Astolfi, "Nonlinear model reduction by moment matching," Foundations and Trends in Systems and Control, vol. 4, no. 3-4, pp. 224-409, 2017.

[11] W. Cummins, "The impulse response function and ship motions," DTIC Document, Tech. Rep., 1962.

[12] J. Falnes, Ocean waves and oscillating systems: linear interactions including wave-energy extraction. Cambridge university press, 2002.

[13] A. Mérigaud and J. V. Ringwood, "Free-surface time-series generation for wave energy applications," IEEE Journal of Oceanic Engineering, 2017.

[14] G. Scarciotti and A. Astolfi, "Moment-based discontinuous phasor transform and its application to the steady-state analysis of inverters and wireless power transfer systems," IEEE Transactions on Power Electronics, vol. 31, no. 12, pp. 8448-8460, 2016.

[15] Y. Peña-Sanchez, M. Garcia-Abril, F. Paparella, and J. V. Ringwood, "Estimation and forecasting of excitation force for arrays of wave energy devices," IEEE Transactions on Sustainable Energy, 2018.

[16] K. Hasselmann, "Measurements of wind wave growth and swell decay during the Joint North Sea Wave Project (JONSWAP)," Dtsch. Hydrogr. Z., vol. 8, p. 95, 1973. 